\title{
MaDaTS: Managing Data on Tiered Storage for Scientific Workflows
}

DOI: $10.21105 /$ joss.00830

\section{Software}

- Review ct

- Repository ca

- Archive ${ }^{\top}$

Submitted: 03 July 2018 Published: 01 October 2018

\section{License}

Authors of papers retain copyright and release the work under a Creative Commons Attribution $4.0 \mathrm{In}$ ternational License (CC-BY).

\section{Devarshi Ghoshal ${ }^{1}$ and Lavanya Ramakrishnan ${ }^{1}$}

1 Lawrence Berkeley National Lab

\section{Summary}

Scientific workflows are processing large amounts of data through complex simulation and analysis tasks. Meanwhile, the need to minimize I/O costs on next generation systems and the evolution of new technologies (NVRAMs, SSDs etc.) is resulting in deeper storage hierarchies on High Performance Computing (HPC) systems. A multi-tiered storage hierarchy introduces complexities in workflow and data management. There is need for simple and flexible data abstractions that can allow users to seamlessly manage workflow data and tasks on HPC systems with multiple storage tiers. MaDaTS (Managing Data on Tiered Storage for Scientific Workflows) provides an API and a command-line tool that allows users to manage their workflows and data on tiered storage (Ghoshal \& Ramakrishnan (2017)).

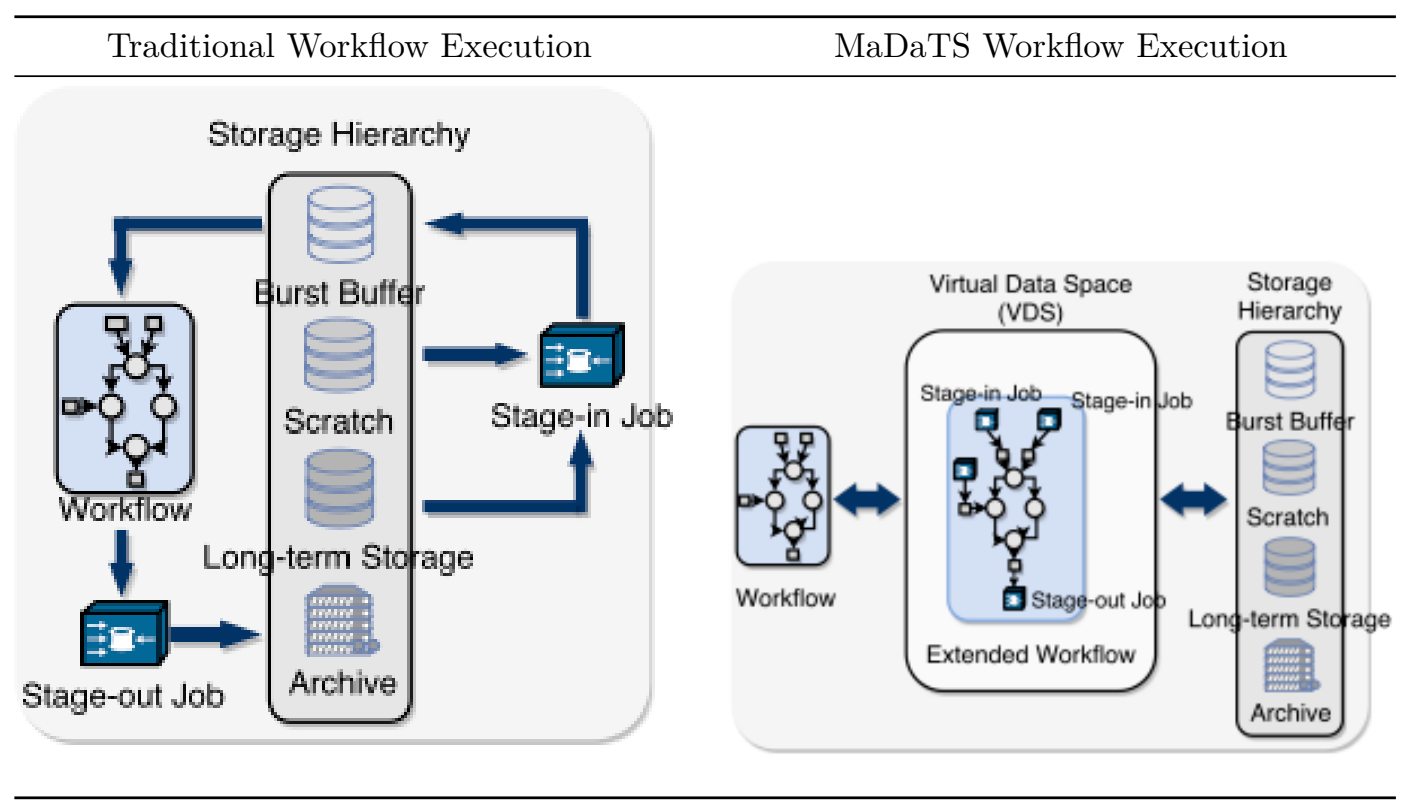

The traditional programming model defines a workflow as a collection of tasks using data as inputs and outputs. This makes data management complex on multi-tiered storage systems because the data needs to be managed explicitly as the workflow tasks execute, ensuring that the data dependencies and consistencies are handled correctly. MaDaTS is built on top of an abstraction called Virtual Data Space (VDS) that hides the complexities of managing data on tiered storage systems. VDS is a collection of virtual data objects. A virtual data object is an abstraction of workflow data in VDS, associated with certain properties and tasks. MaDaTS uses these task associations and properties to define the actual location of data at runtime. In MaDaTS, users simply need to create 
the appropriate virtual data objects and add them to a VDS. MaDaTS takes care of all the data management and workflow execution tasks.

\section{Data Management Abstractions}

MaDaTS provides a simple data management abstraction through the manage() interface. Users create a VDS and tell MaDaTS to manage workflow data and tasks. The 'manage' interface also allows users to group the tasks in different ways. Additionally, users can select the data management strategy in MaDaTS. By default, MaDaTS provides three data management strategies - i) workflow-aware: data management decisions are made based on the structure of the workflow, ii) storage-aware: data management decisions are made based on the storage properties, and iii) passive: allows users to define custom data placement and movement policies.

Users can also define new data management strategies in MaDaTS by defining data tasks through the create_data_task() interface. The interface adds the necessary data operations needed for efficient execution of the workflow. Both workflow-aware and storageaware data management strategies use 'create_data_task' to add supplementary tasks that operate on managing data across the storage tiers. These data tasks are executed as part of the workflow and are managed through MaDaTS.

\section{References}

Ghoshal, D., \& Ramakrishnan, L. (2017). MaDaTS: Managing data on tiered storage for scientific workflows. In Proceedings of the 26th international symposium on highperformance parallel and distributed computing, HPDC '17 (pp. 41-52). New York, NY, USA: ACM. doi:10.1145/3078597.3078611 